\title{
Article
}

\section{Passive and Active Microrheology of Hard-sphere Colloids}

L. G. Wilson, A. W. Harrison, A. B. Schofield, J. Arlt, and W. C. K. Poon

J. Phys. Chem. B, 2009, 113 (12), 3806-3812• DOI: 10.1021/jp8079028 • Publication Date (Web): 09 February 2009

Downloaded from http://pubs.acs.org on April 8, 2009

\section{More About This Article}

Additional resources and features associated with this article are available within the HTML version:

- Supporting Information

- Access to high resolution figures

- $\quad$ Links to articles and content related to this article

- Copyright permission to reproduce figures and/or text from this article

\section{View the Full Text HTML}




\title{
Passive and Active Microrheology of Hard-sphere Colloids
}

\author{
L. G. Wilson,* A. W. Harrison, A. B. Schofield, J. Arlt, and W. C. K. Poon \\ SUPA, COSMIC and School of Physics and Astronomy, The University of Edinburgh, Kings Buildings, \\ Mayfield Road, Edinburgh EH9 3JZ, United Kingdom
}

Received: September 5, 2008; Revised Manuscript Received: December 16, 2008

\begin{abstract}
We performed passive and active microrheology using probe particles in a bath of well-characterized, model hard-sphere colloids in the fluid state over the whole range of volume fractions below the glass transition. The probe and bath particles have nearly the same size. Passive tracking of probe particles yields short-time self-diffusion coefficients. Comparison with literature data demonstrates that the interaction between probe and bath particles is hard-sphere-like. The short-time diffusivities yield one set of microviscosities as a function of volume fraction, which agrees with previous macrorheological measurements of the high-frequency viscosity of hard-sphere colloids. Using optical tweezers, we measure the force on a trapped probe particle as the rest of the sample is translated at constant velocity. This yields a second set of microviscosities at high Péclet numbers. These agree with previous macrorheological measurements of the high-shear viscosity of similar colloids, at shear-rates below the onset of shear-thickening.
\end{abstract}

\section{Introduction}

Microrheologies. Complex fluids contain "mesoscopic" structural elements such as colloidal particles, polymer coils, or self-assembled surfactant aggregates. The long characteristic times associated with the Brownian motion of these mesoscopic entities can give rise to significant viscoelasticity. Understanding the rheology of complex fluids ${ }^{1}$ poses fundamental challenges and has many practical implications. The ubiquity of complex fluids in living systems means that advances in understanding their rheology also has significant implications for biology.

Traditionally, complex fluid rheology is measured in welldefined "rheometric" geometries (cone-plate, Couette, etc.) in a rheometer using macroscopic amounts of sample, minimally $\approx 1 \mathrm{~cm}^{3}$. However, such quantities are often unavailable, especially in newly synthesized materials or biology. In the latter case, in vivo measurements inside cells and/or tissues are often desirable. Increasingly, therefore, microrheological methods are being developed. ${ }^{2}$

In a typical passive microrheology experiment, the fluctuating position of a probe particle, with radius $a$, in a complex fluid is tracked as a function of time, and a generalized Stokes-Einstein relation is used to extract frequency-dependent rheological properties, ${ }^{3}$ typically the storage and dissipative moduli, $G^{\prime}(\omega)$ and $G^{\prime \prime}(\omega)$ (where $\omega$ is the angular frequency). The tracked particle's mean square displacement can also be used to yield a diffusion coefficient directly (which, in general, is timedependent), from which a microviscosity can be deduced, again via a generalized Stokes-Einstein relation.

Passive microrheology has now been validated and extended to a more robust two-particle version ${ }^{4}$ and applied to a variety of materials. ${ }^{2}$ Because the system is being probed under quiescent conditions, passive microrheology yields linear viscoelastic properties.

On the other hand, in active microrheology, a probe particle is dragged through the medium under investigation, and the force acting on the particle, $\vec{F}$, as well as its velocity, $\vec{U}$, are monitored.

* To whom correspondence should be addressed. E-mail: Laurence.Wilson@ed.ac.uk.
In a Newtonian liquid at typical microrheological Reynolds numbers $\left(\operatorname{Re} \leqslant 10^{-5}\right.$ ), Stokes law holds, and measurement of a single pair of $\vec{F}$ and $\vec{U}$ yields the viscosity of the liquid, $\eta_{0}=$ F/6 $\pi a U$.

In a complex fluid, varying $F$ or $U$ permits, in principle, the study of a wide range of linear and nonlinear mechanical response. Controlling $F$ and controlling $U$ in active microrheology are not equivalent. ${ }^{5}$ In a yield-stress material, for example, systematic investigation of yielding is only possible by varying $F$ and not $U$. In either case, whenever there is flow, an effective microviscosity can be defined:

$$
\eta_{\text {micro }}^{\text {(active) }}=\frac{F}{6 \pi U a}
$$

Relating $\eta_{\text {micro }}^{\text {(active) }}$ to viscosities measured in macrorheological experiments is not simple, because the flow field set up by a probe particle being dragged through a medium is highly nonrheometric. ${ }^{6}$

Active Microrheology of Colloids. The microrheology of colloids has been studied theoretically for hard spheres ("bath" particles with radius $b$ ) by Brady and co-workers. 5,7,8 They predict the force-velocity relation of probe particles at different $\alpha=a / b$ both with and without hydrodynamic interactions at low colloid volume fraction $(\phi) .^{7}$ These predictions were confirmed semiquantitatively by simulations at $\alpha=1$, especially for the case of controlled $F{ }^{8}$ Although these results are likely to apply best to dilute charged colloids, a number of points raised by Brady and co-workers have generic validity.

First, a test particle translated at constant $\vec{U}$ is more dissipative than one dragged at constant $\vec{F}$, since it has to push away every encountered particle rather than "go round" it, as is allowed under constant $\vec{F}$. Second, there is a subtlety concerning the Péclet number. The basic dimensionless group is the same in both modes: $\mathrm{Pe}=U(a+b) / D$, where $D$ is the relative diffusion coefficient between probe particle (radius $a$ ) and bath colloid (radius $b$ ), but the latter differs according to mode. The probe particle diffuses under constant $F$ but not under constant $U$. In the case of $\alpha=1$, this leads to 


$$
\begin{gathered}
\mathrm{Pe}_{F}=\frac{F a}{k_{\mathrm{B}} T} \\
\mathrm{Pe}_{U}=\frac{2 U a}{D_{0}}
\end{gathered}
$$

where $k_{\mathrm{B}} T$ is the thermal energy, and $D_{0}=k_{\mathrm{B}} T / 6 \pi \eta_{0} a$ is the free diffusion coefficient of a probe particle in pure solvent. (Note that there is a certain arbitrariness in numerical factors in these definitions. In particular, the definition we adopt for $\mathrm{Pe}_{U}$, eq 3, agrees with that used by Squires and Brady ${ }^{5}$ to interpret their simulation data but differs by a factor of 2 from that used by Carpen and Brady. ${ }^{8}$ ) Third, dragging a probe particle using optical tweezers constitutes a "mixed mode", but if the spring constant $\kappa$ of the optical trap satisfies

$$
\frac{\alpha \kappa(a+b)^{2}}{k_{\mathrm{B}} T} \gg \mathrm{Pe}_{U}
$$

that is, if the probe is trapped strongly relative to the flow forces acting on it, constant $U$ behavior is recovered.

Experimentally, Habdas et al. ${ }^{9}$ have dragged a magnetic bead through suspensions of sterically stabilized polymethylmethacrylate (PMMA) colloids $(\alpha \approx 2 ; 0.29 \leq \phi \leq 0.56)$ under controlled $\vec{F}$ at $100 \lesssim \mathrm{Pe}_{F} \lesssim 10000$. At $\phi=0.29$, they found $F \propto U$ at all Pe and measured a normalized microviscosity of $\eta_{\text {micro }}^{\text {(active) } /} \eta_{0} \approx 2.7 \pm 0.45$. Previous macrorheological measurements for the same kind of particles at this $\phi$ found a normalized macroviscosity of $\eta_{r}^{\text {macro }} \approx 3.2$ in the low-shear limit and $\eta_{r}^{\text {macro }}$ $\approx 2.5$ in the high-shear limit $^{10}$ below the onset of shearthickening, so the value of Habdas et al. is somewhat closer to the high-shear value. This is not unexpected given the very large values of $\mathrm{Pe}_{F}$ involved. At $\phi \geq 0.45$, a threshold force, $F_{0}$, was needed to move the bead, beyond which the average probe velocity grows superlinearly with $F-F_{0}$. (Recent microrheology theory and simulations ${ }^{11}$ suggest that this behavior is due to the experiments not reaching low-enough $\mathrm{Pe}_{F}$. According to these recent results, the observed "threshold" is simply part of an overall shear-thinning response.) Such superlinear relationship implies "force thinning", again consistent with bulk rheology, ${ }^{10}$ but Habdas et al. did not provide any quantitative comparison with bulk rheology at any other volume fractions.

In another set of experiments, Meyer et al. ${ }^{12}$ used optical tweezers to drag polystyrene, silica, or melamine probe particles through an aqueous suspension of Teflon particles $(6 \lesssim \alpha \lesssim$ $20 ; 0.08 \leq \phi \leq 0.31)$ at $3<\mathrm{Pe}_{U}<700$. The choice of Teflon particles was dictated by the need for index matching, which then allowed nonindex-matched probe particles to be trapped optically. Meyer et al. found that their $\eta_{\text {micro }}^{\text {(active) }}$ at the lower end of Péclet numbers studied agreed with the low-shear viscosity measured by bulk rheology, whereas nonlinear $F-U$ behavior at higher Pe was interpreted as shear thinning.

The PMMA colloids studied by Habdas et al. ${ }^{9}$ have been extensively characterized over more than two decades and are known to behave as model hard spheres. ${ }^{13}$ Much less information is available about the Teflon particles used by Meyer et al. ${ }^{12}$ in particular, they are only quasi-spherical, and the interparticle interaction is unknown. Meyer et al. claimed that their macrorheology data were consistent with the Teflon particles behaving like hard spheres. However, they measured $\eta_{r}^{\text {macro }} \gtrsim 8$ at $\phi=0.3$, whereas measurements of the bestcharacterized hard sphere colloids in the literature (sterically stabilized PMMA) repeatedly gave $\eta_{r}^{\text {macro }} \approx 3$ at both the lowand high-shear limit for $\phi \approx 0.3 \cdot{ }^{10,14}$ (Compare also the result of Habdas et al., cited above.) The viscosity of the Teflon particles used by Meyer et al. was therefore growing with $\phi$ much more rapidly than hard spheres, indicating possible interparticle attraction. In both sets of experiments, ${ }^{9,12}$ the interaction between the probe and bath particles was uncharacterized.

In this work, we report passive and active microrheology measurements of PMMA colloids in the entire range of volume fractions below the glass transition $\left(\phi<\phi_{g} \approx 0.58\right)$. The colloids are index- and buoyancy-matched to the solvent. Indexmismatched and sterically stabilized melamine particles are used as probes. Passive tracking of the probe particles yield the shorttime self-diffusion coefficient, $D_{\text {short }}^{\text {self }}$ which, when compared with very extensive literature data, shows that the probes interact with the bath particles in the same way as bath particles interact with one another. This data also yield one set of microviscosities, $\eta_{\text {micro }}^{\text {(passive) }}$. Active rheological measurements at high Péclet numbers are then performed by trapping probe particles using optical tweezers and translating the sample relative to the trap. This yields another effective viscosity, $\eta_{\text {micro }}^{\text {(active) }}$. We compare these microviscosities with previous macrorheological measurements. Our results provide a baseline data set for understanding the microrheology of a very well-characterized model hard-sphere system.

\section{Materials and Methods}

Particles. We used PMMA particles sterically stabilized by chemically grafted poly-12-hydroxystearic acid (PHSA), synthesized following literature procedures. ${ }^{15}$ The particles were dispersed in a mixture of mixed (cis- and trans-) decalin and cycloheptylbromide (CHB) to achieve density matching at room temperature (checked by prolonged centrifugation). This solvent mixture also closely matches the refractive index of the particles $(n=1.49)$. It is known that CHB induces charges on PMMA particles. ${ }^{16}$ Following previous work, we added $1 \mathrm{mg} / \mathrm{cm}^{3}$ tetrabutylammonium chloride to screen out the charge and recover model hard-sphere behavior. The viscosity of the suspending medium was measured (in an AR2000 Rheometer, TA Instruments) to be $\eta_{0}=2.56 \mathrm{mPa} \cdot \mathrm{s}$.

Two batches of particles were used, with radii in the densitymatching medium determined (by light scattering) to be $b=$ 860 and $960 \mathrm{~nm}$ respectively. At high volume fractions, these particles do spontaneously crystallize, albeit very slowly (over the course of months); their polydispersity is thus close to but just below $\approx 12 \% .{ }^{17}$ We have never observed crystallization during experimentation in situ. Samples of different volume fractions were prepared by diluting and resuspending the sediment formed by spinning in a centrifuge away from the density-matching temperature, and assuming a volume fraction of 0.64 for this sediment. Suspensions were sealed between standard microscope coverslips for observation.

Following Meyer et al., ${ }^{12}$ we dope the index-matched bath colloids with index-mismatched tracer particles. The latter were made of melamine resin $(n=1.7)$ and were purchased from Microparticles $\mathrm{GmbH}$ with radius and polydispersity quoted as $a=950 \mathrm{~nm}$ and 5\%, respectively. We coated the melamine particles with PHSA to ensure that they interact with the bath PMMA particles in the same way as the bath particles interact among themselves. To this end, ethanol-dispersed melamine particles were mixed with a solution of PHSA in tetrahydrofuran. The mixture was well-stirred and then heated to remove the solvents. Decalin was simultaneously added to keep the particles dispersed. To monitor successful adsorption, the PHSA stabilizer 
backbone was tagged with the fluorescent polymerizable NBDMMA dye. ${ }^{18}$ In a confocal microscope, the coated particles showed a ring of fluorescence (data not shown). Below we show data demonstrating that the PHSA-coated melamine particles interact with the PMMA colloids as hard spheres. We aimed to study a system in which probe and bath particles were as nearly indistinguishable as possible. Dynamic light scattering gave a radius of $a=1.04 \mu \mathrm{m}$ to our coated melamine particles, so that $\alpha=1.2$ and 1.08 for our two bath particle batches. We found no systematic difference between data obtained using these two batches of particles within our experimental uncertainties. We therefore do not distinguish between data from these two batches.

Optical Setup. We implemented standard optical tweezers in a home-built microscope. ${ }^{19}$ An Nd:YAG laser (operating at $\lambda=1064 \mathrm{~nm}$ ) was used both for trapping the probe particle and sensing its position, the latter via imaging onto a quadrant photodiode (QPD). ${ }^{20}$ The setup is calibrated using welldocumented standard procedures. ${ }^{21,22}$ Briefly, we record the fluctuating voltage output from the quadrant photodiode (at 5 $\mathrm{kHz}$ ) due to the motion of the image of a trapped probe particle in quiescent solvent. The power spectrum of the latter should be a Lorentzian:

$$
S(f)=\frac{D_{0}}{\pi^{2}\left(f_{\mathrm{c}}^{2}+f^{2}\right)}
$$

where $D_{0}$ is the free diffusion coefficient of the particle, and the "corner frequency", $f_{\mathrm{c}}$, is given by

$$
f_{\mathrm{c}}=\frac{\kappa}{2 \pi \xi_{0}}
$$

with $\xi_{0}=6 \pi \eta_{0} a$, so that $D_{0}=k_{\mathrm{B}} T / \xi_{0}$. The derivation of this result is available elsewhere, ${ }^{23}$ but its physical interpretation is clear. At high frequencies (or short times), the particle essentially diffuses freely, and its power spectrum scales as

$$
S(f)=\frac{D_{0}}{\pi^{2} f^{2}} \text { when } f \gg f_{\mathrm{c}}
$$

The saturation of the power spectrum at $f<f_{c}$ reflects the saturation of the real-space mean-squared displacement due to the optical trap. A Lorentzian fit to the power spectrum of the QPD output (Figure 1), which is proportional to particle displacement in the focal plane in the limit of small displacement, yields the trap stiffness $\kappa$ (typically $\sim 10^{-6} \mathrm{~N} / \mathrm{m}$ ) via the corner frequency and the conversion factor relating QPD output voltage to particle displacement (typically $10^{-3} \mathrm{~V} / \mathrm{nm}$ ). We note that this procedure requires $\eta_{0}$ and $a$ as input. Indeed, the accuracy to which these quantities are known turns out to be the limiting factor in determining the final uncertainties in our results.

For displacements $z a / 2$, the optical potential as well as QPD response become nonlinear. To calibrate in this range, we translated the microscope stage at a fixed velocity and recorded the displacement of the trapped probe particle in pure solvent simultaneously using the QPD and a CCD camera that was itself first calibrated by imaging a precision slide. Direct comparison yielded the conversion factor from QPD output to particle displacement, whereas the optical potential was obtained by

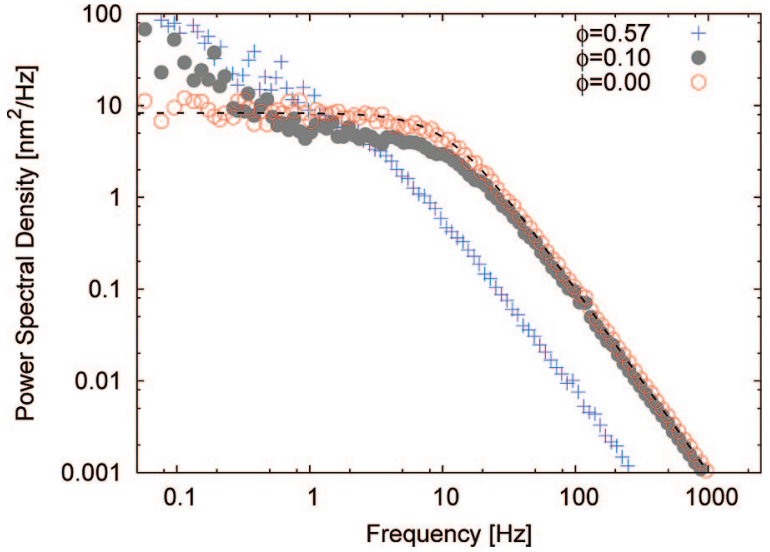

Figure 1. The power spectrum of the fluctuating position of a trapped melamine particle in pure solvent $(\square)$ and in a bath of PMMA colloids at volume fractions $\phi=0.1(\bullet)$ and $\phi=0.57(+)$. Dashed line $=$ Lorentzian fit, eq 5, to the free-particle data, from which we calibrate our optical trap for position and force measurements.

calculating the drag force on the particle via Stokes law. The potential scales linearly with the incident laser power.

A DC motor was used to translate the stage. The highest accessible $\mathrm{Pe}_{U}$ is limited by the fluctuations in position of the trapped particle, which start to extend beyond the boundary of the optical trap. The lowest accessible Pe is set by our motor, which had a lowest speed of $100 \mathrm{~nm} / \mathrm{s}$. Like previous work using optical tweezers, ${ }^{12}$ our data span just over two decades in Pe.

During our measurements, the probe particle is trapped at some 20 particle radii from the nearest wall in the sample compartment. At this distance, the wall changes the hydrodynamic drag on the probe from the Stokes law value by a few percent, ${ }^{24}$ which is negligible compared to other sources of experimental uncertainty. We therefore neglect such "Faxén corrections" in our data analysis. A typical run for data collection lasted $\sim 15 \mathrm{~min}$.

\section{Microrheological Measurements}

Passive. Equation 5 is valid in the limit of a single trapped particle in pure solvent. At even the lowest concentration of bath colloids we studied, $\phi=0.1, S(f)$ is already non-Lorentzian at low frequencies, Figure 1, but eq 5 remains valid in the limit $f \gg f_{\mathrm{c}}$, that is, the particle motion remains free diffusion at short enough times. Thus, at all $\phi$, fitting the high-frequency part of $S(f)$ allows us to measure the short-time self-diffusion coefficient of the probe particle, $D_{\text {short }}^{\text {self }}(\phi)$. We can extract a microviscosity from the short-time self-diffusivities according to the generalized Stokes-Einstein relation:

$$
\frac{\eta_{\text {micro }}^{\text {(passive) }}(\phi)}{\eta_{0}}=\frac{D_{0}}{D_{\text {short }}^{\text {self }}(\phi)}
$$

We report our measurements of $D_{\text {short }}^{\text {self }}$ in terms of this microviscosity in Figure 2. The main contribution to the error bars shown is the $5 \%$ polydispersity in the melamine radius used in calibrating the optical tweezers. This underestimates the expected data scatter. Because we only average measurements of a few (typically 3 or 4 ) particles at each $\phi$, it is always possible that one or more of the chosen particles are far from the mean size used in calibration calculations. Diffusion in PMMA suspensions has been measured in detail using dynamic 


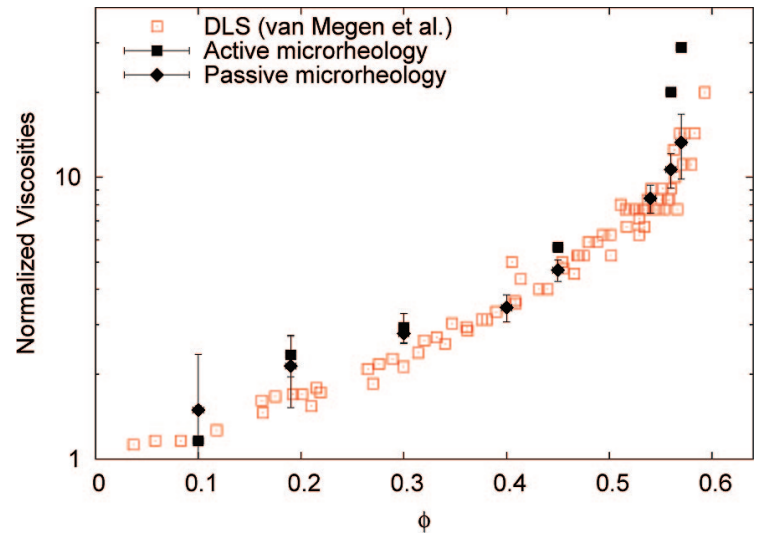

Figure 2. The microviscosities of PMMA suspensions normalized to the solvent viscosity measured using passive and active probe techniques: $\eta_{\text {micro }}^{\text {(passive) }} / \eta_{0}(\diamond)$ and $\eta_{\text {micro }}^{\text {(active) }} / \eta_{0}(\mathbb{\square})$. The microviscosity deduced from light scattering measurements of the short-time self-diffusion coefficient ${ }^{26}$ is also plotted ( $\square$ ). The horizontal axis gives the colloid volume fraction $\phi$.

light scattering (DLS), and the results were found to fit theoretical predictions and computer simulations for hard spheres up to $\phi \lesssim 0.5 .{ }^{25}$ The short-time diffusion coefficient of tracers in this system measured by DLS should be directly comparable to our passive data, except that by automatically averaging over many more particles, DLS delivers superior statistics. The best current literature DLS data for $D_{\text {short }}^{\text {self }}(\phi)$ (Figure 4 in van Megen's work), ${ }^{26}$ reported as a normalized microviscosity (i.e., $\left.D_{0} / D_{\text {short }}^{\text {self }}\right)$, are shown alongside our data in Figure 2. Within experimental uncertainties, the two methods agree, such agreement being particularly convincing at $\phi \gtrsim 0.4$, where uncertainties in the probe size in our measurements contributes a smaller absolute error bar on the diffusivity.

The short-time self-diffusion coefficient is determined by the direct and hydrodynamic interactions between the tracer and its neighbors and is a sensitive function of local environment, particularly at high $\phi$. In the case of hard spheres, the only relevant interactions are hydrodynamic at these short time scales. The agreement between our measurements and DLS data suggest that the PHSA chains coating our melamine particles have rendered them hard-sphere-like as far as the bath PMMA colloids are concerned.

Active. We next translated the sample stage at fixed velocity, $\vec{U}$, relative to the laser trap, and measured the displacement of the trapped (probe) particle in the imaging plane, $\vec{r}$, as a function of time. The prior calibration of the trap then allows us to calculate the drag force, $F$, from the measured displacements. The advantage of such active microrheology is that not only is the average force-speed response, $\langle F(U)\rangle$, available, but also full information on fluctuations, $F(t)$. These fluctuations, which increase with $U$, can be analyzed to give interesting information on basic statistical mechanical issues relating to various nonequilibrium "work theorems". ${ }^{27}$ Such analyses will be published elsewhere. ${ }^{28}$ Here we concentrate on the average drag force on the probe particle when it is translating at fixed speed $U$, and report our results in terms of a microviscosity, $\eta_{\text {micro }}^{\text {(active) }}=\langle F(U)\rangle /$ $6 \pi a U$ as a function of $\mathrm{Pe}_{U}=2 U a / D_{0}$, eq 3 . The main source of uncertainties in these results is the randomly shifting zero of the trap position due to inherent mechanical noise. Our results are shown in Figure 3.

At each of the two lowest concentrations studied $(\phi=0.1$ and 0.19$), \eta_{\text {micro }}^{\text {(active) }}$ is essentially constant over the whole range of $\mathrm{Pe}_{U}$ we accessed. At the next two concentrations, $\phi=0.30$

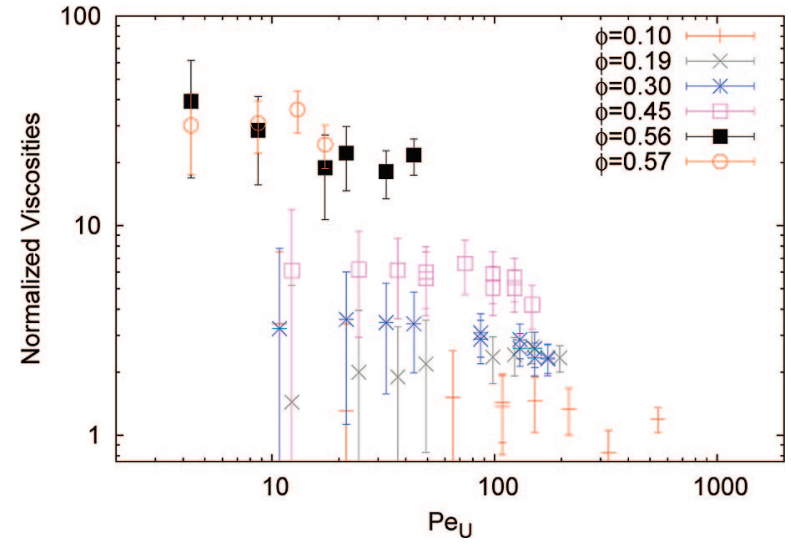

Figure 3. The normalized active microviscosity, $\eta_{\text {micro }}^{\text {(active) }} / \eta_{0}$, as a function of constant- $U$-Péclet number for various volume fractions, $\phi$.

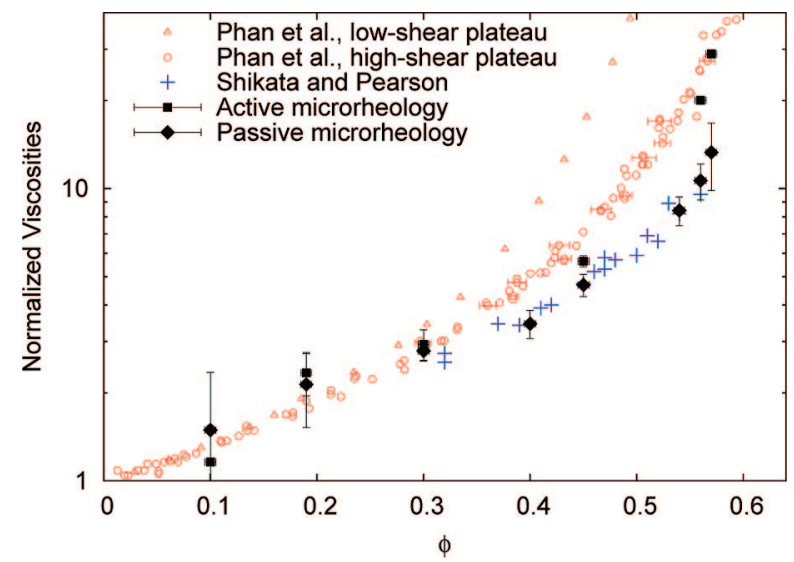

Figure 4. Various macro- and microviscosities for PMMA colloids as a function of volume fraction. This work: $\eta_{\text {micro }}^{\text {(passive) }} / \eta_{0}(\diamond)$ and $\eta_{\text {micro }}^{\text {(active) }} /$ $\eta_{0}(\square)$. Literature macrorheology data: low-shear $(\Delta)$ and high-shear ( $\square$ ) viscosities in PMMA colloids from Phan et al. ${ }^{10}$ and high-frequency viscosities $(+)$ in silica colloids interacting as hard spheres from Shikata and Pearson, ${ }^{29}$ all normalized to the solvent viscosity $\eta_{0}$. The horizontal axis gives the colloid volume fraction $\phi$.

and $0.45, \eta_{\text {micro }}^{\text {(active) }}$ is apparently constant over the lower end of our range of $\mathrm{Pe}_{U}$, but rather abruptly "dips" to lower values at the highest values of $\mathrm{Pe}_{U}$ reached. Such dips can also be seen in the data of Meyer et al. ${ }^{12}$ Data for the highest two concentrations, $\phi=0.56$ and 0.57 , are again consistent with no $\mathrm{Pe}_{U}$ dependence.

Meyer et al. suggested that the dips in their data were due to "shear thinning". We argue later that such dipping could also be an instrumentation artifact due to the probe particle exploring the "edge" of the optical trap. For now, we disregard the points associated with the dips at intermediate $\phi$ and average the data at different values of $\mathrm{Pe}_{U}$ for each $\phi$ to arrive at an estimate of another microviscosity, $\eta_{\text {micro }}^{\text {(active) }}$ the results are shown in Figure 2.

\section{Discussion}

Figure 2 shows that the microviscosities obtained using passive and active methods, $\eta_{\text {micro }}^{\text {(passive) }}$ and $\eta_{\text {micro }}^{\text {(active) }}$, are indistinguishable within experimental uncertainties at low volume fractions, $\phi \lesssim 0.3$. On the other hand, at higher $\phi, \eta_{\text {micro }}^{\text {(active) }}$ is systematically higher than $\eta_{\text {micro }}^{\text {(passive) }}$.

To begin to make sense of our findings, we first turn to literature data for macroviscosities of hard-sphere suspensions. 
We have plotted in Figure 4 measurements by Phan et al. of the low-shear and high-shear viscosities of PMMA suspensions, ${ }^{10} \eta(\phi)$ and $\eta_{\infty}^{(\dot{\gamma})}(\phi)$, respectively, as well as Shikata and Pearson's data $^{29}$ for the high-frequency viscosity of silica colloids interacting as hard spheres, $\eta_{\infty}^{(f)}(\phi)$. These three macroviscosities are distinct, satisfying $\eta(\phi)>\eta_{\infty}^{(\dot{\gamma})}(\phi)>\eta_{\infty}^{(f)}(\phi)$. It appears that our passive microviscosity closely follows literature data for the high-frequency macroviscosity, $\eta_{\text {micro }}^{\text {(passive) }} \approx \eta_{\infty}^{(f)}(\phi)$, whereas the active microviscosity tracks the macroviscosity $\eta_{\text {micro }}^{(\text {active })} \approx \eta_{\infty}^{(\dot{\gamma})}(\phi)$ at shear-rates that are high, but below the onset of shear-thickening.

Passive Microrheology. Consider first $\eta_{\text {micro }}^{\text {(pasive) }}$, which is deduced from $D_{\text {short }}^{\text {self }}$. Because the short-time self-diffusivity probes the high-frequency "jiggling" of the probe particle in an almost-fixed cage of neighbors, we have a priori reason to expect $\eta_{\text {micro }}^{\text {(pasive) }}$ to be comparable to $\eta_{\infty}^{(f)}$. However, there is no reason to expect identity; in other words, there is no theoretical reason to expect the generalized Stokes-Einstein relation, eq 8 , to be valid.

Observationally, however, $\eta_{\text {micro }}^{\text {(passive) }}$ appears to agree with literature values of $\eta_{\infty}^{(f)}(\phi)$ measured for silica particles interacting as hard spheres to within experimental uncertainties for $\phi \gtrsim$ 0.3 . This apparent near-identity should be treated with caution. Simulations suggest that

$$
\frac{\eta_{\infty}^{(f)}}{\eta_{0}} \frac{D_{\text {short }}^{\text {self }}}{D_{0}} \approx 1+0.75 \phi
$$

for $\phi \lesssim 0.45 .^{30}$ In other words, we should expect the macrorheology values $\eta_{\infty}^{(f)}$ to be systematically higher than the values of $\eta_{\text {micro }}^{\text {(pasive) }}$ derived from $D_{\text {short }}^{\text {self }}$, with the difference increasing at increasing $\phi$. A $\phi$-dependent relationship between diffusion and viscosity is also expected on theoretical grounds. ${ }^{31}$ This is not observed for the data sets plotted in Figure 4.

One reason may be the inherent uncertainties in comparing different data sets. Shikata and Pearson also reported the viscosity of their system in the zero frequency limit, $\eta(\phi) .{ }^{29}$ Their data do not agree with that reported for PMMA hard spheres, ${ }^{10,14}$ unless the volume fractions for the silica data are multiplied by a factor of 0.9 . Such a shift of the $\eta_{\infty}^{(f)}$ data set in Figure 4 would give qualitative agreement with eq 9 . However, we must also recall that the silica and PMMA systems had different polydispersities. Such issues mean that detailed comparison to the level of accuracy required to test eq 8 is not possible with currently available data.

Active Microrheology. To interpret our active microrheology results, we first need to understand the time scales behind the relevant Péclet number, $\mathrm{Pe}_{U}$, eq 3. A natural time scale in our system is the Brownian time, $\tau_{\mathrm{B}}$, the time it takes a particle in pure solvent to diffuse its own radius. For a particle of radius $a$,

$$
\tau_{\mathrm{B}}=\frac{a^{2}}{6 D_{0}}=\frac{\pi \eta_{0} a^{3}}{k_{\mathrm{B}} T}
$$

Another time scale is the lifetime of a particular "cage" of neighbors surrounding a particle, $\tau_{\text {cage. }}$ This natural "cage rearrangement" time can be determined, for example, using mean-squared displacements (MSDs) measured from DLS. The MSD is diffusive at short times (the slope being $D_{\text {short }}^{\text {self }}$ ), subdiffusive at intermediate times, and returning to diffusive at long times (the slope being the long-time self-diffusivity). The point of inflection in the MSD vs time plot may be taken as a measure of $\tau_{\text {cage. Interestingly, in the PMMA system, it was }}$ found $^{26}$ that $\tau_{\text {cage }} \approx 4 \tau_{\mathrm{B}}$ up to $\phi \approx 0.5$, before diverging rapidly at higher $\phi$. Moreover, the root mean squared displacement at $\tau_{\text {cage }}, \Delta$, a measure of "cage size", can be fitted to $\Delta / a=$ $2.2-3.6 \phi$.

In a constant- $U$ active microrheology experiment, we should compare the time it takes the dragged probe particle to travel $\Delta, \tau_{\text {probe }}=\Delta / U$, with $\tau_{\text {cage. }}$. If $\tau_{\text {probe }} \gg \tau_{\text {cage }}$, then the probe does not need to "break cages", and we are in the "low speed" regime. On the other hand, if $\tau_{\text {probe }} \ll \tau_{\text {cage }}$, then the probe is actively breaking cages, and we are in the "high speed" regime. The crossover happens when $\tau_{\text {probe }} \approx \tau_{\text {cage }}$. Using the above-reported results for $\tau_{\text {cage }}$ and $\Delta^{26}$ and eq 3 , we predict the crossover $\mathrm{Pe}_{U}$ for our system to occur at

$$
\mathrm{Pe}_{U}^{\text {crit }} \approx 6.6-10.8 \phi
$$

for $\phi \lesssim 0.5$. Thus, all of our data should be in the high-Pe $\mathrm{P}_{U}$ regime. (Note that the rapid divergence of $\tau_{\text {cage }}$ above $\phi=0.5$ leads to values of crossover $\mathrm{Pe}_{U}$ significantly below that predicted by eq 11.)

In macrorheology, the relevant Péclet number for controlled shear rate $(\dot{\gamma})$ experiments is $\operatorname{Pe}_{\dot{\gamma}}=6 \pi \eta_{0} a^{3} \dot{\gamma} /\left(k_{\mathrm{B}} T\right)$. This dimensionless number measures the relative importance of particle rearrangements due to Brownian motion and shear. Again, at high $\mathrm{Pe}_{\gamma}$, cages are broken by shear rather than rearranged by Brownian motion. Thus, we may expect macroand active microrheology data to be comparable at high values of $\mathrm{Pe}_{\gamma}$ and $\mathrm{Pe}_{U}$, respectively. This makes sense of our observation, Figure 4 , that $\eta_{\text {micro }}^{\text {(active) }}$ tracks $\eta_{\infty}^{(\dot{\gamma})}(\phi)$.

However, as in the case of passive measurements, we have no theoretical reason to expect the observed near-identity of these two viscosities, Figure 4. Again, this result must be interpreted with caution. Although the particles in our experiments and in those of Phan et al. ${ }^{10}$ are both sterically stabilized PMMA dispersed in very similar solvents, the colloids had different polydispersities. Moreover, the volume fraction was measured differently in the two investigations: we normalized to random close packing, which we take to be $\phi=0.64$, whereas Phan et al. normalized to the crystallization transition of a slighly polydisperse system of hard spheres found in simulations. We have shown before ${ }^{14}$ that small uncertainties in volume fractions can translate into large uncertainties in viscosities at high enough volume fractions $(\eta \gtrsim 0.5)$.

Because we do not have data in the low- $\mathrm{Pe}_{U}$ regime, we cannot test eq 11 directly. It predicts critical Péclet numbers somewhat bigger than the critical $\mathrm{Pe}_{\dot{\gamma}}$ for silica particles in macrorheology, ${ }^{32}$ although the agreement is significantly better at $\phi \gtrsim 0.3$. We may also compare our results with the Brownian dynamics simulations of Carpen and Brady at $\alpha=1 .^{8}$ Although the majority of their simulations were performed at constant force, these authors also showed a single data set at constant probe velocity for $\phi=0.35$. They found that $\eta_{\text {micro }}^{\text {(active) }} / \eta_{0}$ decreased from $\approx 2.1$ in the limit of $\mathrm{Pe}_{U} \rightarrow 0$ to a value of $\approx 1$ in the limit of $\mathrm{Pe}_{U} \rightarrow \infty$, with the "thinning" becoming noticeable at $\mathrm{Pe}_{U} \approx$ 2. (Note that we have multiplied the $\mathrm{Pe}_{U}$ values in the simulations of Carpen and Brady ${ }^{8}$ by 2 , as the choice of $\mathrm{Pe}_{U}$ in that work differs from ours). Experimentally, we find $\eta_{\text {micro }}^{\text {(active) }} / \eta_{0}$ $\lesssim 3$ at $\phi=0.3$. This means that the simulations underestimate the high-Pe $\mathrm{Pe}_{U}$ microviscosity; but this is to be expected, since hydrodynamic interactions were neglected. However, the critical value for "velocity thinning" in the simulation, $\mathrm{Pe}_{U}^{\text {crit }} \gtrsim 2$, is in 
reasonable agreement with eq 11 , which predicts a value of $\mathrm{Pe}_{U}^{\text {crit }}$ $\approx 2.8$ for $\phi=0.35$.

Finally, we return to the matter of the dip in the intermediate- $\phi$ data sets in Figure 3. Meyer et al. ${ }^{12}$ argued that similar dips in their data were due to the operation of a thinning mechanism unique to active microrheology. ${ }^{6}$ Although this is possible, these dips could also be instrumental artifacts. At low $\phi$, the probe particle is always within the linear region of the optical trapping potential. As the $\phi$ increases, the drag force on the probe particle rises and it starts to explore the outlying, anharmonic parts of this potential. Here, the force constant becomes progressively weaker, eventually reaching zero. Although we have calibrated and corrected for this nonlinearity in converting displacement to force, the decreasing stiffness at the edges of the potential has another effect that is harder to quantify. (If such correction is not made we will overestimate the force, and therefore the viscosity, at high $\mathrm{Pe}$, giving a rise in the data.)

As the probe particle increasingly explores the softening edges of the trap at high $\phi$, there is an increasing probability of losing the probe from the trap. Since we discarded position traces from particles that were 'lost' during data taking, we may have a systematic bias toward lower than average displacements, and so return microviscosities that are too low at intermediate $\phi$. We worked with approximately constant increments in probe speed, which correspond to progressively larger steps in drag force between data points as $\phi$ increases. At the highest volume fractions, it is likely that we step directly from the last data point taken in the linear region of the trapping potential to beyond the escape force, thus bypassing the nonlinear region all together, so that no dips are seen.

\section{Summary and Conclusion}

To summarize, we have probed well-characterized hardsphere colloidal fluids at a range of volume fractions below the glass transition using passive and active microrheology and have measured two microviscosities as functions of volume fraction. The passive microviscosity closely follows literature data for the high-frequency macroviscosity, although the active microviscosity at high $\mathrm{Pe}_{U}$ tracks the nonshear-thickened, high-shearrate macroviscosity.

In our experiments, $\alpha \gtrsim 1$, and we operated at either high $f$ or high $\mathrm{Pe}_{U}$; the observed near-identity in each case is unexpected. It is possible that discrepancies, for example, as predicted by eq 9, are masked by uncertainties arising from differences in polydispersity and $\phi$ calibration in the data sets contributing to Figure 4. Future work using both micro- and macro-techniques on the same sample may clarify this point. On the other hand, there may be special reasons in hard spheres why micro- and macroviscosities should track each other closely in the high-frequency and high-shear limits. This would be analogous to the previous finding that in the same PMMA system the collective diffusion coefficient measured at the wavevector corresponding to the peak of the structure factor has the same volume fraction dependence as the low-shear viscosity up to $\phi \approx 0.5$ to within experimental uncertainties. ${ }^{33}$ Subsequent theory shows that there should only be an approximate equality, and no such relationship holds in charged colloids. $^{34}$

We considered microscopic processes involving cage breaking to estimate the critical value of $\mathrm{Pe}_{U}$ separating high and low probe velocity regimes in active microrheology, eq 11. Such discussion suggests a role for direct imaging. Meyer et al. ${ }^{12}$ have published images of the bath suspension during the passage of a probe particle, showing large "wakes" qualitatively similar to simulation results. ${ }^{8}$ The bath particles used by Meyer et al. were too small to resolve optically, but in the system used by Habdas et al., ${ }^{9}$ and indeed that used in this work, both probe and bath particles can be imaged directly. Confocal microscopy can be used to build up a threedimensional picture both in the quiescent system $^{35}$ and to study probe-driven rearrangements ${ }^{36}$ with single-particle resolution. ${ }^{37,38}$ Future experiments of this kind will give insight into the microstructural processes contributing to measured microviscosities. Moreover, we have worked in volume fractions below the glass transition. Microrheological experiments near ${ }^{9}$ and within the glassy regime can yield significant and unique information on the glass transition. Many intriguing theoretical predictions, such as "negative differential mobility" 39 (the decrease in a probe particle's mobility when it is forced through its surroundings), are yet to be tested experimentally. Thus, the "simple" experiment of dragging a probe particle through a colloidal suspension contains multiple layers of complexity, the exploration of which has only just begun.

Acknowledgment. This work is funded by EPSRC grants EP/E030173/1 and EP/D071070/1 and two EPSRC studentships. We thank Rut Besseling, Gary Bryant, Mike Cates, Antonio Puertas, Peter Pusey, and Thomas Voigtmann for helpful discussions and Rut Besseling for technical assistance.

\section{References and Notes}

(1) Larson, R. The Structure and Rheology of Complex Fluids; Oxford University Press: Oxford, 1999.

(2) Waigh, T. A. Rep. Prog. Phys. 2004, 68, 685-742.

(3) Mason, T. G. Rheol. Acta 2000, 39, 371-378.

(4) Levine, A.; Lubensky, T. C. Phys. Rev. Lett. 2000, 85, 1774-1777.

(5) Squires, T. M.; Brady, J. F. Phys. Fluids 2005, 17, 073101.

(6) Squires, T. M. Langmuir 2008, 24, 1147-1159.

(7) Khair, A. S.; Brady, J. F. J. Fluid Mech. 2006, 557, 73-117.

(8) Carpen, I. C.; Brady, J. F. J. Rheol. 2005, 49, 1483-1502.

(9) Habdas, P.; Schaar, D.; Levitt, A. C.; Weeks, E. R. Europhys. Lett. 2004, 67, 477-483.

(10) Phan, S.-E.; Russel, W. B.; Cheng, Z.; Zhu, J.; Chaikin, P. M.; Dunsmuir, J. H.; Ottewill, R. H. Phys. Rev. E 1996, 54, 6633-6645.

(11) Gazuz, I.; Puertas, A. M.; Voigtmann, Th.; Fuchs, M., preprint arXiv:0810.2627.

(12) Meyer, A.; Marshall, A.; Bush, B. G.; Furst, E. M. J. Rheol. 2006, $50,77-92$.

(13) Underwood, S. M.; Taylor, J. R.; van Megen, W. Langmuir 1994, 10, 3550-3554.

(14) Poon, W. C. K.; Meeker, S. P.; Segrè, P. N.; Pusey, P. N. J. NonNewton. Fluid Mech. 1996, 67, 179-189.

(15) Antl, L.; Goodwin, J. W.; Hill, R. D.; Ottewill, R. H.; Owen, S. M; Papworth, S.; Waters, J. A. Colloids Surf. 1986, 17, 67-78.

(16) Yethiraj, A.; van Blaaderen, A. Nature 2003, 421, 513-517.

(17) Pusey, P. N. J. Phys. (Paris) 1987, 48, 709-712.

(18) Jardine, R. S.; Bartlett, P. Colloid Surface A 2002, 211, 127-132.

(19) Methods in Cell Biology: Laser Tweezers in Cell Biology; Sheetz, M. P., Ed.; Academic Press: San Diego, 1998.

(20) Gittes, F.; Schmidt, C. F. Opt. Lett. 1998, 23, 7-9.

(21) Bartlett, P.; Henderson, S. J. Phys.: Condens. Matter 2002, 14, $7757-7768$.

19.

(22) Berg-Sørensen, K.; Flyvbjerg, H. Rev. Sci. Instrum. 2004, 75, $1-$

(23) Gittes, F.; Schmidt, C. F., Chapter 8 in ref 19.

(24) Neuman, K. C.; Block, S. M. Rev. Sci. Instrum. 2004, 75, 2787.

(25) Segrè, P. N.; Behrend, O. P.; Pusey, P. N. Phys. Rev. E 1995, 52, 5070-5083.

(26) van Megen, W. Phys. Rev. E 2006, 73, 011401.

(27) Kurchan, J. J. Stat. Mech. 2007, 7, P07005.

(28) Puertas, A.; Wilson, L. G., Poon, W. C. K. in preparation.

(29) Shikata, T.; Pearson, D. S. J. Rheol. 1994, 38, 601-616.

(30) Ladd, A. J. C. J. Chem. Phys. 1990, 93, 3884-3894.

(31) Fuchs, M.; Mayr, M. R. Phys. Rev. E 1999, 60, 5742-5751.

(32) van der Werf, J. C.; de Kruif, C. G. J. Rheol. 1989, 33, 421-454. 
(33) Segrè, P. N.; Meeker, S. P.; Pusey, P. N.; Poon, W. C. K. Phys. Rev. Lett. 1995, 75, 958-961.

(34) Banchio, A. J.; Nägele, G.; Bergenholtz, J. J. Chem. Phys. 1999, $111,8721-8740$.

(35) Prasad, V.; Semwogerere, D.; Weeks, E. R. J. Phys.: Condens. Matter 2007, 19, 113102.

(36) Besseling, R.; Isa, L.; Weeks, E. R.; Poon. W. C. K., Adv. Colloid Interface Sci. . preprint arXiv:0807.4705.
(37) Jenkins, M. C.; Egelhaaf, S. U. Adv. Colloid Interface Sci. 2008 , $136,65-92$.

(38) Besseling, R.; Isa, L.; Weeks, E. R.; Poon, W. C. K. Adv. Colloid Interface Sci. 2008, doi: 10.1016/j.cis.2008.09.008.

(39) Jack, R. L.; Kelsey, D.; Garrahan, J. P.; Chandler, D. Phys. Rev. E 2008, 78, 011506 .

JP8079028 